\title{
Myoepithelioma of the lacrimal gland: report of a case with spindle cell morphology
}

\author{
Ramon L Font, Alec Garner
}

\begin{abstract}
The case is described of a 23-year-old female patient presenting with unilateral proptosis, headaches, and transient epiphora. Surgery revealed an encapsulated tumour composed exclusively of spindle-shaped cells within a richly vascularised myxoid stroma. Immunohistochemical staining showed focal positivity for smooth muscle actin, vimentin, and glial fibrillary acidic protein. These combined findings are interpreted as providing evidence of a myoepithelioma, which may be regarded as a monomorphic adenoma consisting solely of myoepithelial cells. To our knowledge this is only the second report of such a tumour in the lacrimal gland.
\end{abstract}

(Br F Ophthalmol 1992; 76: 634-636)

It may be that most benign adenomatous tumours of the lacrimal gland are derived from the myoepithelial component of the parenchyma. These cells differentiate to produce unmistakeable epithelial and, frequently, overtly glandular structures as well as, in the vast majority of instances, a variably hyaline, myxoid, or chondroid extracellular stroma. The resultant tumour is then aptly named a pleomorphic adenoma, and in this respect the lacrimal gland parallels its salivary counterpart. Occasional salivary gland adenomas, however, are monomorphic in that fully differentiated ductular or duct-derived epithelium alone is seen, while even more unusually the tumours are restricted to a myoepithelial proliferation with an absence of manifest glandular components. Monomorphic adenomas arising in the lacrimal gland are still rarer: tumours with a uniform glandular pattern have yet to be described and there has been only one previous report of a pure myoepithelial proliferation. ${ }^{1}$ Here we describe a second case.

\section{Case report}

Department of Pathology, Institute of Ophthalmology, London A Garner

Department of Ophthalmology, Cullen Eye Institute, Baylor College of Medicine, Houston, Texas R L Font

Correspondence to: Professor A Garner, Institute of Ophthalmology, 17-25 Cayton Street, London ECIV 9AT.

Accepted for publication 9 April 1992
A 23-year-old woman presented to the orvitat clinic of Moorfields Eye Hospital complaining of gradually increasing proptosis of her left eye. She had been conscious of some 'puffiness' around the eye for up to 1 year and for the last 3 months had suffered occasional left parietal headaches and transient epiphora. Ophthalmic examination revealed $5 \mathrm{~mm}$ proptosis with $2-3 \mathrm{~mm}$ downward displacement of the left eye and there are some restriction of eye movement on upgaze, particularly in abduction. Visual acuity was not affected (RE 6/6; LE 6/4) and the ocular fundi were ophthalmoscopically normal. A mass could be palpated in the left lacrimal fossa and ultra-

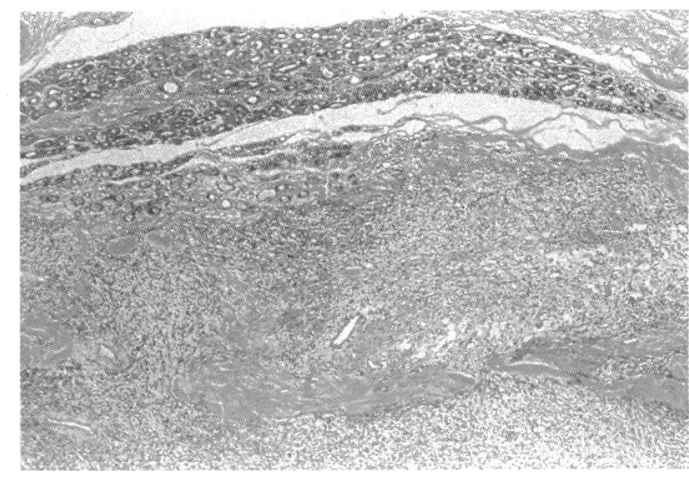

Figure 1 Histological section showing elongated lobules of atrophic lacrimal gland tissue adjacent to the capsule of the tumour (haematoxylin and eosin, $\times 32$ ).

sonography produced abnormal echoes temporal to the globe, both superiorly and inferiorly.

The preoperative clinical diagnosis was lacrimal gland tumour, probably benign, and 7 weeks. later a circumscribed and apparently encapsulated mass was excised through a left lateral orbitotomy. There was no detectable involvement of the adjacent periosteum and the postoperative course was uneventful.

At the present time, more than 12 years after surgery, the patient is alive and well with no sign of recurrent tumour.

\section{Pathology}

Grossly, the resected tumour showed a smooth, encapsulated, pale grey, firm and reniform mass measuring approximately $30 \times 25 \times 17 \mathrm{~mm}$. Microscopically, the mass was covered by a fibrous capsule enclosing a solid tumour composed exclusively of spindle-shaped cells Lobules of atrophic lacrimal gland tissue were confined to one edge of the tumour adjacent to its capsule (Fig 1). Most of the tumour cells had rounded or elongated nuclei according to the

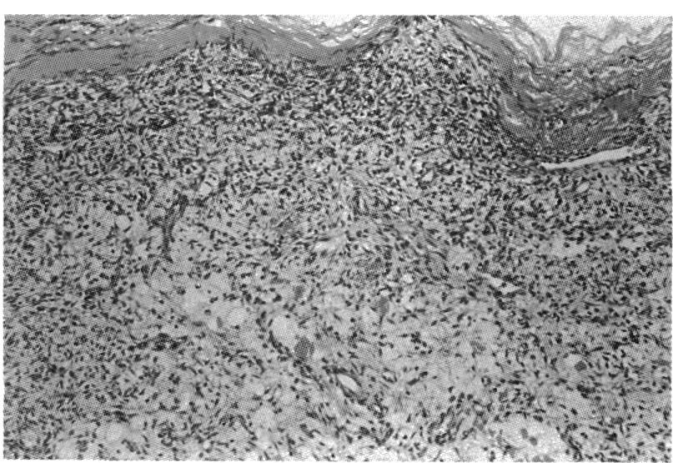

Figure 2 The lacrimal gland mass is covered by a fibrous capsule and consists of spindle-shaped cells intermixed with numerous capillaries (haematoxylin and eosin, $\times 80$ ). 


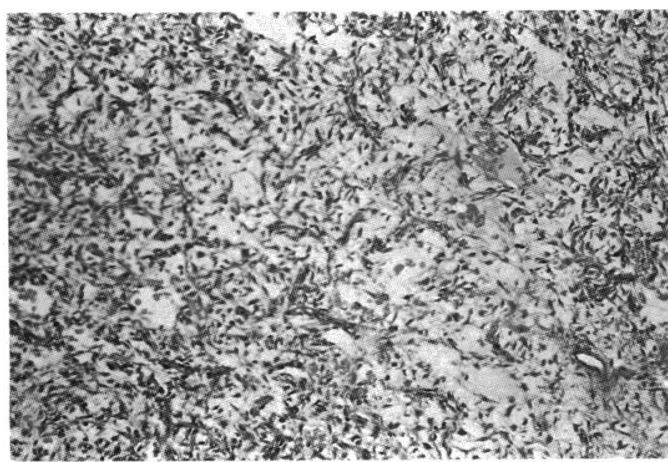

Figure 3 The centre of the tumour is similarly composed of spindle-shaped cells with thin, elongated, cytoplasmic processes embedded in a myxoid matrix rich in capillaries (haematoxylin and eosin, $\times 80$ ).

cellular orientation with inconspicuous nucleoli but occasionally slightly enlarged and hyperchromatic nuclei were also seen. Mitotic figures were rare. The cell cytoplasm was either eosinophilic or vacuolated, especially in less densely populated parts of the tumour where a prominent myxoid stroma was observed (Figs 2 and 3). A rich capillary network with scattered large sinusoidal vessels was present. In some areas the tumour displayed confluent cystoid spaces containing proteinaceous exudate and occasional erythrocytes (Fig 4). The loose myxoid areas alternated with dense aggregates of spindle cells, some of which demonstrated a perivascular arrangement (Fig 5). Areas of necrosis with secondary haemorrhage were present and in places this appeared to have been superseded by hyaline fibrous tissue.

Immunohistochemical procedures using a peroxidase-antiperoxidase method demonstrated focal response of some cells with antibodies against smooth muscle actin, vimentin, and glial fibrillary acidic protein (GFAP). Labelled antibody to low molecular weight keratin (AE1) stained the residual lacrimal ducts adjacent to the capsule of the tumour but the spindle cells were unreactive (Fig 6). Negative staining reactions were obtained using antibodies to $S-100$ protein.

\section{Discussion}

It is a common finding that a proportion of pleomorphic adenomas in both the salivary ${ }^{2}$ and the lacrimal ${ }^{3}$ glands contain solid foci of myoepithelial cell proliferation. The validity of a

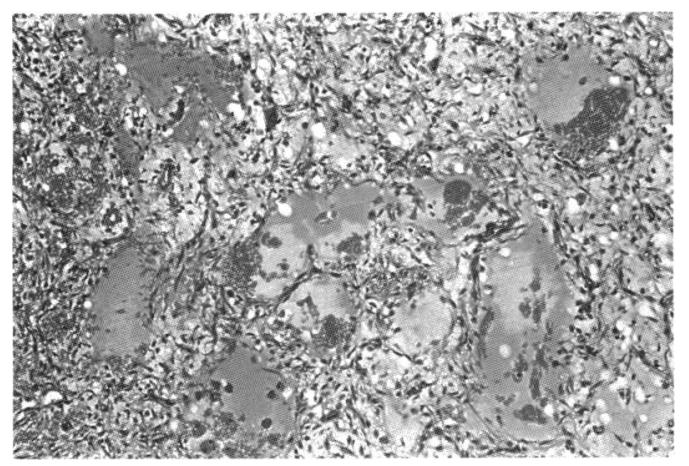

Figure 4 In other areas the mass exhibits cystoid spaces containing proteinaceous exudate and erythrocytes (Masson trichrome, $\times 80$ ).

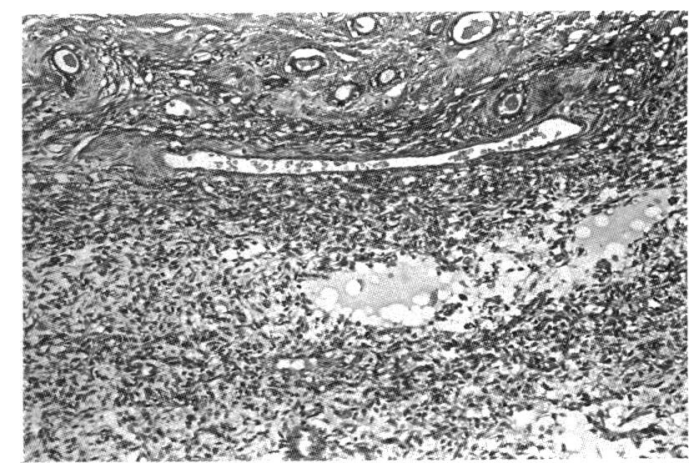

Figure 5 The capsule of the tumour seen in the upper part of the photomicrograph, includes several lacrimal gland ducts, while the underlying tumour shows cohesive perivascular cells alternating with loose areas consisting of proteinaceous exudate (Masson trichrome, $\times 80$ ).

diagnosis of myoepithelioma, however, depends on demonstrating that the tumour is essentially monomorphic and that the constituent cells are myoepithelial. While this can reasonably be construed from the shape of the cells in the present case, the diagnosis is rendered more secure by the evidence that a proportion of the spindle-shaped cells contained smooth muscle actin. Grossniklaus and colleagues, ${ }^{+}$in an immunohistochemical study of normal and neoplastic lacrimal gland tissue, found that myoepithelium in situ at the perimeter of normal secretory acini reacts strongly with antisera to smooth muscle actin, rarely with antibodies to GFAP and strongly but inconsistently with a wide spectrum of antikeratin antibodies. The same study showed that a minority of the spindle-shaped stromal cells in pleomorphic adenomas were positive for smooth muscle actin, while rare stromal cells stained weakly for vimentin. In their study, positive reactions for keratin and GFAP were associated with a proportion of so-called epithelioid as opposed to spindle-shaped myoepithelial cells. A weak response for GFAP was the sole positive immunohistochemical staining seen in the other case of lacrimal gland myoepithelioma. 'It would seem, however, that caution must be used in the interpretation of such data. Thus, although GFAP reactivity has been used as a criterion for the identification of myoepithelial cells, it was positive in only two of 23 normal salivary glands in one extensive study. ${ }^{5}$ Similarly, S-100 protein

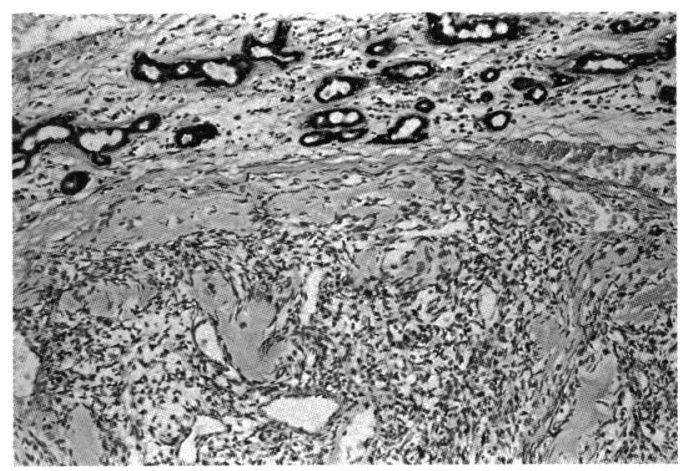

0
0
0
0
0
0
0
0
0
0
0
0
0
0
0
0
0
0
0
0
0
0
0
0
0
0
0
0
0
0
0
0
0
0
0
0
0
0
0
0
0
0
0
0
0
0
0
0
0
0
0
0
0
0
0
0
0
0
0
0
0
0
0
0
0

Figure 6 Section of an area adjacent to that seen in Figure 5 showing lacrimal gland ducts immunoreactive for cytokeratin (brown-black), in contrast to the underlying spindle cells which are negative (low molecular weight keratin AE $1, \times 80$ ). 
the S-100b form of which has formerly been regarded as a marker for myoepithelium, ${ }^{6}$ has more recently been shown to be confined to closely associated autonomic nerves within the glands. ${ }^{7}$ That being the case, the negative reaction for S-100 protein in both our patient and that of Heathcote and colleagues ${ }^{1}$ is not contrary to a diagnosis of myoepithelioma.

Ultrastructural characteristics of myoepithelial cells, such as micropinocytotic vesicles, cytoplasmic filaments consistent with actin and a pericellular basement membrane, have been described in myoepithelial tumours of the salivary glands ${ }^{8}$ and in the one recorded lacrimal gland example of this type of tumour. ${ }^{1}$ We believe, however, that these are not pathognomonic features and are not essential for the diagnosis and note that Dardick and others ${ }^{8}$ defined myoepitheliomas solely on the basis of their light microscopic and immunohistochemical properties. We contend, therefore, that it is reasonable to regard the present tumour as a spindle-cell myoepithelioma and, despite the finding of occasional cells with abnormal nuclei, to have anticipated a benign prognosis borne out by the patient's 12-year trouble-free postoperative survival.

The potential for a variety of adenoma types, ranging from pure ductal proliferations (monomorphic type) through lesions of mixed cell type to pure myoepithelial tumours, to be seen in the lacrimal gland is readily appreciated on the assumption that, as has been reasoned in the context of the salivary glands, ${ }^{5}$ they all originate from a common precursor. Whether or not that precursor is myoepithelial, as has generally been assumed, is not clear because of inconsistent and potentially ambiguous immunohistochemical marker findings. It is also reasonable to assume that most adenomas will occupy the mid-range of the spectrum and be mixed tumours, though it might be wondered why the extremes are encountered less frequently in the lacrimal gland than in the salivary glands. Using the salivary tumours as a guide, however, there is no cause to regard monomorphic adenomas, including myoepitheliomas, as having a different biological behaviour from the more usual pleomorphic lesions. 9 This is a point of some importance because myoepitheliomas involving the salivary glands have quite often been diagnosed as malignant tumours, ${ }^{10}$ as for a time was the present case. As applied in the salivary gland context, a number of morphologically different subtypes of myoepithelioma are described with $90 \%$ consisting of spindle-shaped or polygonal cells. ${ }^{8}$ We believe that both Heathcote and colleagues' case and ours, are examples of pure spindle cell myoepithelial tumours. Additionally, there is a third case recorded by Rootman ${ }^{11}$ which we suspect represents the same entity.

We are grateful to Mr John Wright, Moorfields Eye Hospital, for access to the clinical information.

1 Heathcote JG, Hurwitz JJ, Dardick I. A spindle-cell myoepithelioma of the lacrimal gland. Arch Ophthalmol 1990; 108: 1135-9.

2 Thackray AC, Sobin LH. Histological typing of salivary gland tumours. Geneva: World Health Organisation, 1972; 9-28.

Ashton $\mathrm{N}$. Epithelial tumours of the lacrimal gland. In: Bleeker GM, Garston JB, Kronenberg B, Lyle TK, eds. Bleeker GM, Garston JB, Kronenberg B, Ly

4 Grossniklaus HE, Abbuhl MF, Mclean IW. Immunohistologic properties of benign and malignant mixed tumor of the lacrimal gland. Am $\mathcal{F}$ Ophthalmol 1990; 110: $540-9$.

5 Stead RH, Qizilbash AH, Kontozoglou T, Daya AD, Riddell RH. An immunohistochemical study of pleomorphic adenomas of the salivary gland: glial fibrillary acidic proteinadenomas of the salivary gland: glial fibrillary acidic protein-
like immunoreactivity identifies a major myoepithelial like immunoreactivity identifies a ma
component. Hum Pathol 1988; 19: 32-40.

6 Hara K, Ito M, Takeuchi J, Iijima S, Endo T, Hidaka H. Distribution of $\mathrm{S}-100 \mathrm{~b}$ protein in normal salivary glands and salivary gland tumors. Virchows Arch [A] 1983; 401: 237-49.

7 Dardick I, Stratis M, Parks WR, DeNardi FG, Kahn HJ. S100 protein antibodies do not label normal salivary gland epithelium. Am f Ophthalmol 1991; 138: 619-28.

8 Dardick I, Thomas MJ, van Nostrand AWP. Myoepithelioma - new concepts of histology and classification: a light and electron microscopic study. Ultrastruct Pathol 1989; 13: 187224.

9 Cho KJ, Kim YI. Monomorphic adenomas of the salivary glands: a clinicopathologic study of 12 cases with immunohistochemical observation. Pathol Res Pract 1989; 184: 614 20.

10 Sciubba JJ, Brannon RB. Myoepithelioma of salivary glands: report of 23 cases. Cancer 1982; 49: 562-72.

11 Rootman J. Disease of the orbit: a multidisciplinary approach. Philadelphia: Lippincott, 1988: 394-405. 